\title{
Direct Digital Manufacturing in the Context of a Circular Economy
}

\author{
Diana A. Fonseca ${ }^{1, a}$, Fábio J.P. Simões ${ }^{1, b}$ \\ ${ }^{1}$ Centre for Rapid and Sustainable Product Development, Rua de Portugal - Zona Industrial \\ 2430-028 - Marinha Grande, Portugal \\ adiana.fonseca@ipleiria.pt, bfabio.simoes@ipleiria.pt
}

\begin{abstract}
Keywords: Direct Digital Manufacturing, Circular Economy, Additive Manufacturing, Materials
\end{abstract}
\begin{abstract}
Additive manufacturing technologies have evolved from prototype to part production. Coupling this shift with organizational transformations evolved rapid prototyping industry into Direct Digital Manufacturing (DDM) industry. Any evolution in production systems is now, more than ever before, dependent on sustainability principles. The development of the DDM industry must be guided by these principles, taking the chance it offers to change the production paradigm. Circular Economy (CE) is a shift in the production and resource management model, and one of the ways to work towards sustainability. Coupling DDM with CE principles is thus contributing to change industrial production to a more sustainable one. This paper intends to relate DDM technologies with CE principles, to identify aspects where DDM is contributing to $\mathrm{CE}$, as well as aspects that should be explored further for that purpose. It is observed that DDM can enable optimization of material and energy usage, modify logistics towards de-localized production and recycling and enable prolonged lifespan of products through better access to spare parts, for instance. It is also observed that DDM has good potential to shift materials usage towards natural materials, especially in a context of consumer or local community production, but not so much within the current industrial context. Education is shown to play a pivotal role, since incorporating circular economy principles in educational contexts should spark a shift in consumer perspectives, modifying demand and hence, industrial production.
\end{abstract}

\section{Introduction}

The manufacturing paradigm has been evolving from mass production, through lean manufacturing, and now towards mass personalization [1][2]. Additive manufacturing (AM) technologies have played an important role in this evolution. Nowadays, the combination of AM technologies, communication networks and software, set up to achieve industrial production of goods and not just rapid prototyping, has lead to the concept of Direct Digital Manufacturing [3][4]. DDM inherits the advantages of AM in terms of design space and expands them towards industrial production, and hence logistics, business models and eventually, even societal transformations.

All this potential must be framed within the scope of sustainability, which is a fundamental aspect for development [5]. Geissdoerfer et al.[6] define sustainability as "the balanced integration of economic performance, social inclusiveness, and environmental resilience, to the benefit of current and future generations". Sustainability is intrinsic of natural systems. Natural resources such as sunlight and water are transformed by organisms and their outputs are successively used downstream without losses by other organisms, until a full cycle is completed. The idea of applying the same basic principle to the life cycle of products was developed by several researchers, under several different scopes, since the 1960's [7]. The concept was named Circular Economy (CE), and has developed since by dedicated institutions (e.g. [8,9]) and moreover, has entered top-level policymakers agenda[10][11]. Although $\mathrm{CE}$ is a widespread term nowadays, its relation to sustainability is sometimes unclear. Generally, they share the same main principles. However, according to Geissdoerfer et al.[6], a CE definition would be "a regenerative system in which resource input and waste, emission, and energy leakage are minimized by slowing, closing, and narrowing material and energy loops. This can be achieved through long-lasting design, maintenance, repair, reuse, remanufacturing, refurbishing, and recycling”. 


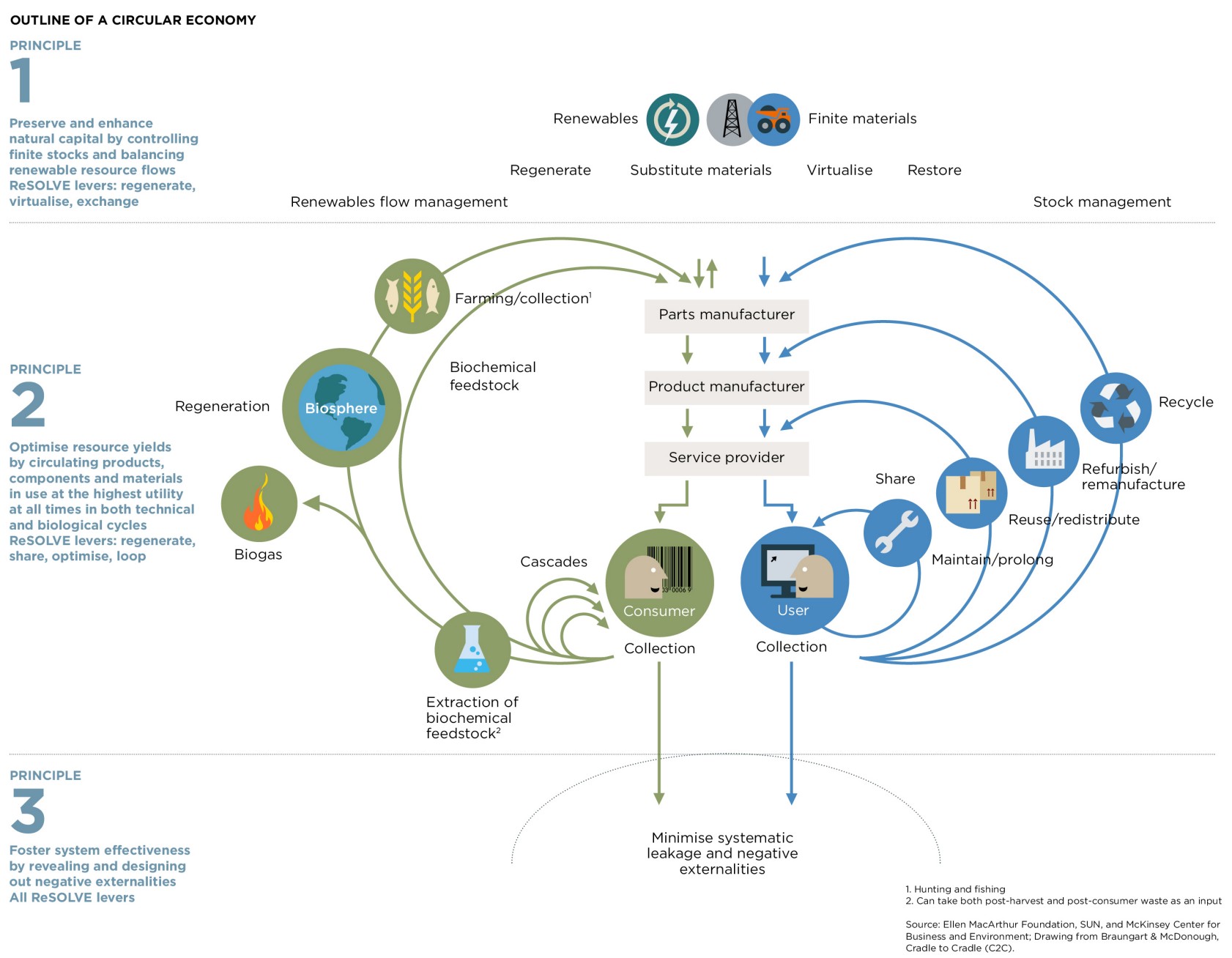

Fig. 1: Circular Economy general perspective[8]

It seems only logical that a technology development having such high potential impact such as AM should enhance the principles of circular economy. However, it is not guaranteed that this will occur [12]. AM technologies were born within the context of a linear economy - "take, make, dispose". Taking advantage of their (relative) early level of development may be one of the ways to effectively enable the transition to the circular economy principles. This review aims to identify the main aspects of AM technologies, and in particular DDM, that can be enhanced towards meeting CE principles. It covers several aspects, identified in previous works ([12][13]), namely materials, design principles, eco-efficiency and logistics.

\section{Direct Digital Manufacturing}

AM technologies were first associated with rapid prototyping. The quality increment made possible by continuous enhancements enables their employment not only for the production of prototypes but for the production of final parts. The advantages of AM technologies in an industrial production context are centred in the original AM technologies advantages, i.e. the expansion of the design space available due to extended geometric freedom, material optimization and reduced assemblies, among others. Tooling becomes unnecessary and thus that cost is eliminated, but the increased cost per part intrinsic to AM technologies means batches will remain low. Moreover, if the aim is exploring AM technologies capabilities of providing customized, on-demand parts, then mass production is out of 
scope for DDM [4]. But DDM has other potential uses, beyond overcoming some of the traditional manufacturing limitations. Being based on digital records, parts can be manufactured in local facilities, instead of being transported [14]. Part records can also be kept to allow spare parts manufacturing long after the product has been discontinued. This may open a change in logistics of many types of industries, as well as business models. Nevertheless, transitioning from AM to DDM implies more than just increasing the quality of parts. For a DDM industry, this implies certification of processes, materials and specialized training of personnel, for instance - [4] citing [15]. This evolution should be encompassed within the principles of Circular Economy, to avoid the pathway of the linear model.

\section{Materials}

AM process was first proposed as mean to build a part using successive layers made of a photosensitive polymer, in a process named stereolithography [16]. Later, other technologies emerged, using inkjet printing principles, powder sintering or binding, laminated object layering, fused deposition [17] and more recently, direct energy deposition [18]. Materials for AM processes evolved towards better surface finish, strength and level of detail [4]. Most widely used materials for AM technologies are photosensitive thermosets, thermoplastics, powder ceramics and powder metals. Composites are also used, especially polymer-matrix composites for fused-deposition modeling - table 1.

Sustainabilty issues When looking at the materials list of table 1 it becomes apparent that most are originated from non-renewable sources. CE principles advocate regenerative systems where resource inputs are minimum. Sustainability needs to be viewed in three aspects: social, economic and environmental. Resource input reduction is an intrinsic part of environmental sustainability. Environmental sustainability can be viewed from the perspective of the source (renewable source, or not), but also from the perspective of material waste minimization, or re-incorporation, especially in the case of non-renewable sources. For AM processes using metal powders, a typical non-renewable resource, reports mention excess material recycling of 95 to $98 \%$ [19], although powder quality will decrease as the material is exposed to repeated heat cycles. In the case of polymer powders, recycling is implemented using sorting systems to preserve powder size and integrity. Ceramic powders have a higher potential for reuse, given their greater low sensitivity to high temperature [4]. Fusion deposition systems use a wide variety of materials, which includes potentially recycled thermoplastics - ABS, PET, PLA, for instance. From these, PLA is the most popular bio-degradable polymer, obtained from renewable (plant) sources, and thus, more sustainable. The others, being oil-derived polymers, are not sustainable, and hence, their sustainability performance will be dependent, at a final level, on their recycling feasibility. Before recycling, parts should, as much as possible, be reused and refurbished figure 1. Recycling is, thus, the last step on a materials loop, in the perspective of CE. It is possible to find initiatives for thermoplastic recycling in the scope of AM technologies, especially within the domestic printers market. Collaborative projects enabled the widespread availability of the technology, and demand for feedstock for fused deposition domestic printers generated initiatives for local recycling of waste plastics to produce filament [20]. Several initiatives can be found aiming to recycle PLA, PET and PE, to produce filament for FDM feedstock. Along with reducing environmental impact, these initiatives are generating new businesses and laying the ground for the decentralization of the recycling systems [20]. Recycling of thermoplastics in an industrial production perspective exists within the scope of recycling of powders in powder-bed fusion processes, as mentioned. For fused deposition processes, industrial printer manufacturers frequently have proprietary feedstock systems, making it harder for the user to recycle materials on his own. Nevertheless, Stratasys ${ }^{\circledR}$ has recycling service for cartridges, canisters, spools, print engines and containers [21]. The reality is still that most industrial providers offer non-sustainable thermoplastics (even though PLA is generally offered) as the main solutions for customers. 
Table 1: AM technologies materials by technology

\begin{tabular}{ll}
\hline Photopolymerization (Stereolithography - SLA) & Epoxy + acrylate resins, Composites \\
\hline Powder bed fusion (PBF) & $\begin{array}{l}\text { Pure metals, Steels, Titanium, nickel and } \\
\text { cobalt alloys, Aluminium alloys, Polyamide, }\end{array}$ \\
& $\begin{array}{l}\text { Polystyrene, Elastomers, PEEK, PCL, PLA, } \\
\text { Aluminium and titanium oxides, Metal-ceramic } \\
\text { composites }\end{array}$ \\
\hline Direct energy deposition (DED) & $\begin{array}{l}\text { Steel, Bronze, Titanium alloys, Nickel alloys, } \\
\text { Cobalt alloys, High-temperature ceramics }\end{array}$ \\
\hline Fused deposition modeling (FDM) & ABS, PLA, PET, PC, Polyamide \\
\hline Material jetting & Waxy Polymers, Acrylic photopolymers, Ce- \\
& ramics suspensions, Solder (electronic circuits), \\
& Aluminium \\
\hline Binder jetting & Ceramics, Plaster, PMMA, Stainless steel, \\
& Nickel alloys, Silica sands, Glass \\
\hline
\end{tabular}

Natural materials. Materials from natural sources seem to be an emerging trend, as yet another consequence of the domestic 3D printer market. Filaments for fused deposition printers made from waste products, incorporating algae, produced from hemp, beer, coffee, waste, flax and others can already be found [22], including flexible filaments [23]. These are said to be compostable, so they fit the renewables flow, as opposed to conventional plastics. Sustainable materials aimed at industrial solutions do not seem to truly be in widespread use yet. However, some filament manufacturers offer "high performance on technical applications, similar to ABS" [24]. From a different perspective, composite materials may provide a significant way to decrease material waste. Referring to CE concepts, incorporating waste particles as reinforcements onto thermoplastic matrices to produce composites, in low demanding applications, can be one of the ways to promote resources re-integration and to close the materials loop, through recycling. In this scope, it is possible to find studies on composites incorporating tyre waste (e.g. [25, 26]), but most importantly, it is possible to identify an increasing trend towards the exploration of natural fibres as reinforcements for composites. Väisänen, Das, and Tomppo [27] have extensively reviewed the advantages, disadvantages and properties of several natural fibres having the potential to be reinforcements on composites. They found that mechanical durability, water absorption, poor thermal properties and processing/production difficulties are still the main disadvantages preventing the application of these natural fibres. Nevertheless, they also identified important potential for improvements, especially by using hybrid animal/plant fibres, who can complement each other in terms of properties. Moreover, it becomes increasingly clear that a sustainable character for both matrices and reinforcements should be an imperative for the development of future materials of this type. Composites such as those described above are also under study for applications on AM processes. For instance, a wood-plastic composite consisting of high-density polyethylene and sawdust was used as feedstock for a large-scale fused deposition process by Horta [28]. It was possible to produce the material by extrusion and to manufacture large-scale parts, although process parameters require further tuning. A fully sustainable solution was still not implemented, even if the polyethylene was originated from waste.

\section{Design}

From a design perspective, AM technologies can contribute for fulfilling several key aspects of CE. The main aspects to achieve this, and their relation to CE principles are summarized on table 2. It is possible to observe that AM technologies can significantly contribute towards CE principles. The main advantages derive from the geometric flexibility and topological optimization. These allow materials savings and performance optimization. 
Examples of AM potential Thompson et al. made an extensive review on design for additive manufacturing [29]. They point out example applications of the performance optimization such as "integrated air ducts and wiring conduits for industrial robots; $3 D$ flexures for integrated actuators and universal grippers; complex internal pathways for acoustic damping devices; optimized fluid channels" but also point out that one of the most significant applications is conformal cooling. This is especially important in injection moulding, where part quality and cycle time can be enhanced simultaneously [30, 31, 32, 33]. Another example is the direct production of assemblies, which reduces manufacturing time, besides being capable of producing parts having joints which would be otherwise impossible to achieve. Generating custom structures towards specific mechanical, thermal, or biological properties is possible by using cellular materials produced by AM. There are numerous examples, especially in the field of regenerative medicine, where specialized FDM printers are used for printing scaffolds for tissue regeneration [34][35]. Using AM processes also enables designing optimized properties for these applications, for instance, elastic modulus [36] [37]. Designing a specific porosity on parts opens up possibilities in the field of biocompatible surfaces for biomedical implants, for increased adhesion. Another aspect derived from geometric flexibility is the customization of parts. DDM can generate customized designs that contribute to the increase of value on each product, extending its life cycle by strengthening the relation of products with theirusers [38][39]. Multi-material parts [40] can also be a part of customization, as well as a part of the optimization of properties, and can contribute to a longer lifespan. Modular designs can be achieved easier with AM technologies, and this is another path to extend a product functionality and lifespan.

Design for AM Widespread adoption of DDM in industrial practice needs the traditional methods of Design Theory and Methodology (DTM), including Design for X - Manufacturing, Assembly, Disassembly and Performance (DFM, DFA, DFD, DFP) to be adapted [45]. Principles of Design for X are:

1. the ease of manufacturing;

2. the ease of assembly;

3. the ease of repair, maintenance, reuse and recycle;

4. manufacture cost reduction;

5. assembly cost reduction;

6. disassembly effort reduction. 
Table 2: AM technologies relation to CE principles.

CE principle AM technology potential

Examples

Optimize resource yields by circulating products, components, and materials at the highest utility at Geometry flexibility towards optimized functionality and perforall times mance

Optimization of objects topology

Direct production of assemblies

Custom surfaces, textures, and porosity for improved functionality

Custom material properties trough structuring

Metamaterials

Waste incorporation
Extending product life and optimizing reuse
Cost effective production of custom-fit and mass customized products

Multi-material parts and products Modular products

Spare parts

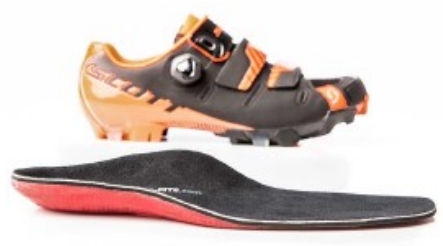

3D printed sole for biking from Tailored Fits AG [38]

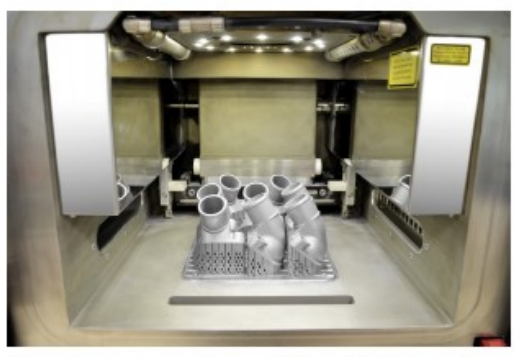

Spare parts for older MercedesBenz vehicles produced by SLM $[43,44]$

In this scope, DDM widespread adoption can contribute to items 1 and 4 almost directly. Assemblies can also be reduced, if not eliminated. As a consequence, disassemblies will be reduced as well. Item 3 is probably not so straightforward. It also becomes apparent that this set of principles misses out on 
some advantages of AM technologies, such as the possibility of using multi-materials or gradients in material properties for increased functionality. Because of this, Yang and Zhao analyze design methods in the scope of AM and identify as main research topics [45]:

- refresh the perspective of traditional design method, bound by manufacturing constraints, and start by imposing functionality requirements;

- do not dismiss assemblies and traditional manufacturing processes; find improved ways to conglomerate them during the design stage;

- develop a mathematical model for multi-functional optimization, facilitating the design process.

AM technologies can break some of the boundaries imposed by these principles, but not all. Additionally, manufacturing does not need to be limited to AM technologies; in fact, hybrid processes (additive + subtractive) are now emerging as an even more flexible approach [46].

Limitations and unexplored aspects of DDM One of the aspects on which the contribution of DDM is still unclear is the design for reuse, remanufacturing, refurbishing, and recycling. Reuse is perhaps the most straightforward aspect that DDM can deliver if the part is designed for increased lifespan. However, DDM cannot accomplish this on its own; a social change, through education, is most definitely needed. At the same time, one or more of previous aspects, especially multi-materials and direct production of assemblies, may not be the best approach when prioritizing refurbishing or recyclability of a product. All of the above implies that design of such parts, while exploring advantages of AM technologies, should take into consideration CE principles first.

\section{Logistics}

The shift on production focus The development of DDM, the design and the production can now be two separate entities. It's starting to be possible to produce locally a part that was designed in somewhere else. This concept opens new ways of looking at logistic chains and production sites. The "3D makers" community is the more obvious facet of this new form of producing goods, although the focus of this community is not on the production by itself, so much as on the empowering of people to take production into their hands. This was one of the principles of the RepRap project [47], which sparked the trend after FDM patent expired. Nowadays, people around the world are designing parts and sharing them freely via Internet sites (e.g. www.thingiverse.com, among others). Everyone can produce a part from the 3D model in the comfort of their home. This networked knowledge has been changing the concept of consumer. The consumer is now not just a passive subject that buys the final product. Now, people can design a new part or modify a 3D model they downloaded to make a part suit their needs. Industrial actors are also joining this trend, e.g. 3dcontentcentral.com, 3dhubs.com, enabling customers to access files for technical parts, customize and produce them by themselves, or to order their production from suppliers. This shift in paradigm from passive consumer to producer/consumer is closing the gap between suppliers and consumers, while promoting local production. These changes, sparked by the hobbyist/enthusiast community, can be extended to all society in the context of circular economy.

Enhanced equipment lifespan One of the aims of circular economy is to maximize the lifespan of a product. One of the ways to achieve this is if spare parts for an equipment are cheap and easy to get. DDM by itself can be an efficient technology for producing spare parts because it can still produce one single spare part for an equipment economically, even if that equipment has been discontinued [44]. Another advantage is in the logistics associated with spare parts. Some companies, like Festo ${ }^{\circledR}$, already have 3D models of their products freely available [48] making it possible for consumer to 
download the part they need and produce locally without the need for transportation or packaging cost. The consumer can also design the part itself (or generate the geometry using a 3D scanner) and make modifications that improve the lifespan of the part. If people start to make spare parts and share them with the community, in the future it will be possible to have a repository of spare parts that people can access, download and produce to repair their pieces of equipment. In this spirit, some pieces of equipment could also be upgraded with new functions, thereby extending their life. Spare parts could be manufactured in a local small or medium enterprise, or even at home, with proprietary or open source hardware.

Local materials and waste management The decentralization of production could lead to the decentralization of materials supply chains. It's important to understand that, in the perspective of CE, formation of new material supply chains can lead to a more sustainable way of producing. These new chains could promote the use of local natural materials, local recycling facilities for the waste generated in that community, and savings in transportation of the materials [12]. With the decentralization of production, people will be more aware of the waste that is generated by a given process. This could encourage the search for new ways of incorporate that waste into new products or increase the demand for products that are more sustainable. Another potential benefit that DDM can promote in the supply chain is the just-in-time production [14]. With DDM it's possible to produce on demand and therefore avoiding obsolete stocks and storage costs. With the reduction of this costs and energy, it's possible, in the future, that DDM products get cheaper.

\section{Education}

Education for sustainability Education is of the six priority areas of Article 6 of the United Nations Framework Convention on Climate Change (UNFCCC). It is usually referred to as Action for Climate Empowerment (ACE) [49] It is becoming increasingly important, as proven by the inclusion within other frameworks, namely the Sustainable Development Goals (SDGs), the Global Action Programme on Education for Sustainable Development (GAP on ESD). The latter took place from 2005 to 2014, "with the goal of emphasizing education in all its forms (formal, nonformal and informal) as an indispensable element for achieving sustainable development" [49]. This global policy trend indicates the need not only to raise awareness on climate change but to empower people to take their own actions toward positive changes.

Education in a circular economy Education plays a pivotal role in a circular economy. A new skill set is required for people transition from the traditional product design, business and consumer models towards CE principles. Governments should be aware of this need and encourage the incorporation of CE principles in all levels of education [8]

3D print communities as informal schools In this scope, using AM technologies as an education tool can help accelerate several aspects of learning related to CE. The appearance of the "makers" community based on open-source AM technologies has promoted sharing between members and created a network of knowledge. This network made possible for everyone to share their knowledge and learn from each other, creating an informal learning environment, that encompasses not only 3D printing but also design and development of hardware [47, 50]. With the development of 3D printing open source hardware, people are able to learn about mechanics, programming and get the comfortable with dealing with some of the processes involved in manufacturing a part. As this community grows, new open source hardware, not limited to 3D printing can be found e.g.[51, 52, 53] 
Relation with CE Making manufacturing technologies available to people empowers them. They can now take charge of producing some of the objects they need, in a similar way as a home garden helps to produce some of their food. It will also raise their awareness of resources and waste management. Moving production and waste management to localities can help reduce costs and improve efficiency. This is an opportunity for education institutions to act in their local community by implementing projects related to the development of local materials, the reincorporation of waste, or repair of products for increased lifespan [54]. Education will empower citizens to rethink their environment and identify ways to act on it. Creativity can be boosted and even new business models can emerge when the design, manufacturing and repair of products are moved locally. The perspective of quality in a product has the potential to change, as people start valuing sustainability and durability over aesthetics only, for instance. Communities will then be better equipped to find the best ways to act towards a circular economy.

\section{Conclusion}

DDM technologies have a massive potential to be the main vehicle in the shift of the manufacturing paradigm from a linear model economy to a circular model economy. This shift, as a sustainability aspect, is no longer considered optional. DDM technologies still rely on linear economy models in many aspects, perhaps the most obvious one being the materials. Sustainability is yet to be the main focal point of industry when choosing materials for manufacturing. This is one of the aspects that require urgent change. Natural materials/composites have potential for playing a key role in this scope. AM technologies are now widespread thanks to the "makers" communities around the world. These communities have an important role in empowering people to take manufacturing in their hands. But this also raised awareness on the other manufacturing-related aspects, such as waste. Now it is possible to find open-source solutions for recycling of filament from 3D prints, for instance. This evolution and enhanced awareness may motivate consumers to demand industrial producers to have their products comply with sustainability principles. The importance and potential of DDM are thus linked both with an industrial production context and with a local, community or consumer-level production, both existing complementary to provide products in a sustainable manner. Education is the vehicle that can convey this change.

\section{Acknowledgements}

This research work was supported by the Portuguese Foundation for Science and Technology (FCT) through the Project references UID/Multi/04044/2013 and PAMI - Portuguese Additive Manufacturing Initiative - CENTRO-01-0145-FEDER-022158.

\section{References}

[1] S. Jack Hu. "Evolving paradigms of manufacturing: From mass production to mass customization and personalization”. In: Procedia CIRP 7 (Jan. 2013), pp. 3-8. issn: 22128271. doi: 10.1016/j.procir.2013.05.002.

[2] Ashok Kumar. "From mass customization to mass personalization: A strategic transformation". In: International Journal of Flexible Manufacturing Systems 19.4 (Dec. 2007), pp. 533-547. issn: 09206299. doi: 10.1007/s10696-008-9048-6.

[3] Danfang Chen et al. "Direct digital manufacturing: Definition, evolution, and sustainability implications". In: Journal of Cleaner Production 107 (Nov. 2015), pp. 615-625. issn: 09596526. doi: 10.1016/j.jclepro.2015.05.009. 
[4] Ian Gibson, David Rosen, and Brent Stucker. Additive Manufacturing Technologies. New York, NY: Springer New York, 2015. isbn: 978-1-4939-2112-6. doi: 10.1007/978-1-4939-2113- 3.

[5] United Nations. Transforming our world: the 2030 Agenda for Sustainable Development. Sustainable Development Knowledge Platform. 2017. url: https://sustainabledevelopment. un.org/post2015/transformingourworld.

[6] Martin Geissdoerfer et al. "The Circular Economy - A new sustainability paradigm?" In: Journal of Cleaner Production 143 (2017), pp. 757-768. issn: 09596526. doi: 10 . 1016 / j . jclepro.2016.12.048.

[7] David Ness. The Emergence of the Modern Circular Economy. 2017. url: http : / www . productlife.org/en/circular-economy.

[8] The Ellen MacArthur Foundation. Circular Economy - UK, USA, Europe, Asia, South America - The Ellen MacArthur Foundation. url: https://www.ellenmacarthurfoundation.org/.

[9] The Product-Life Institute. The Product-Life Institute | Cradle to Cradle. url: http://www. productlife.org/en.

[10] EU Commission. Towards a circular economy: A zero waste programme for Europe. 2014. doi: 10.1017/CBO9781107415324.004. url: http://eur-lex.europa.eu/resource.html? uri $=$ cellar:aa88c66d-4553-11e4-a0cb-01aa75ed71a1.0022.03/DOC1 format=PDF

[11] EU Comission. Circular Economy Strategy - Environment - European Commission. 2017. url: http://ec.europa.eu/environment/circular-economy/.

[12] Mélanie Despeisse et al. "Sustainable Value Roadmapping Framework for Additive Manufacturing”. In: Procedia CIRP 61 (2017), pp. 594-599. issn: 2212-8271. doi: 10.1016/j.procir. 2016.11.186.

[13] Samuel H. Huang et al. "Additive manufacturing and its societal impact: A literature review". In: International Journal of Advanced Manufacturing Technology 67.5-8 (2013), pp. 1191-1203. issn: 02683768. doi: 10.1007/s00170-012-4558-5.

[14] C Tuck, R J M Hague, and N D Burns. "Rapid manufacturing impact on supply chain methodologies and practice". In: International Journal of Services and Operations Management 3.1 (2007), pp. 1-22. doi: 10.1504/IJSOM.2007.011459.

[15] B Hastings. "The transition from rapid prototyping to direct manufacturing". In: SME RAPID Conference. Detroit, 2007.

[16] Jean-Claude Andre. Fr2567668 - Dispositif Pour Realiser Un Modele De Piece Industrielle. 1986. url: http://bases-brevets.inpi.fr/fr/document/FR2567668.html.

[17] J.-P. Kruth, M.C. Leu, and T. Nakagawa. "Progress in Additive Manufacturing and Rapid Prototyping”. In: CIRP Annals - Manufacturing Technology 47.2 (Jan. 1998), pp. 525-540. issn: 00078506. doi: 10.1016/S0007-8506(07)63240-5.

[18] J. Lawrence. Advances in laser materials processing : technology, research and applications. CRC Press, 2010, p. 828. isbn: 9781845694746.

[19] Vojislav Petrovic et al. "Additive layered manufacturing: Sectors of industrial application shown through case studies”. In: International Journal of Production Research 49.4 (2011), pp. 10611079. issn: 00207543. doi: 10.1080/00207540903479786. 
[20] M. A. Kreiger et al. "Life cycle analysis of distributed recycling of post-consumer high density polyethylene for 3-D printing filament". In: Journal of Cleaner Production 70 (2014), pp. 90- 96. issn: 09596526. doi: 10.1016/j.jclepro.2014.02.009.

[21] Recycling | Stratasys. url: http://www.stratasys.com/recycling.

[22] C2renew. c2renew. url: https://www.c2renew.com/materials.

[23] Willowflex. WillowFlex | BioInspiration. url: https://bioinspiration.eu/willowflex/.

[24] Extrudr. BDP biodegradable 3D printing Filament - extrudr - FD3D GmbH. url: https : //extrudr.eu/collections/bdp.

[25] Maciej Sienkiewicz et al. "Environmentally friendly polymer-rubber composites obtained from waste tyres: A review". In: Journal of Cleaner Production 147 (2017), pp. 560-571. issn: 09596526. doi: 10.1016/j.jclepro.2017.01.121.

[26] S Ramarad et al. "Waste tire rubber in polymer blends: A review on the evolution, properties and future”. In: JOURNAL OF PROGRESS IN MATERIALS SCIENCE 72 (2015), pp. 100-140. doi: 10.1016/j.pmatsci.2015.02.004.

[27] Taneli Väisänen, Oisik Das, and Laura Tomppo. “A review on new bio-based constituents for natural fiber-polymer composites". In: Journal of Cleaner Production 149 (2017), pp. 582-596. issn: 09596526. doi: 10.1016/j.jclepro.2017.02.132.

[28] J F Horta, F J P Simões, and A Mateus. "Large scale additive manufacturing of eco-composites". In: International Journal of Material Forming (June 2017). issn: 1960-6214. doi: 10.1007/ s12289017-1364-5.

[29] Mary Kathryn Thompson et al. "Design for Additive Manufacturing: Trends, opportunities, considerations, and constraints”. In: CIRP Annals - Manufacturing Technology 65.2 (2016), pp. $737-$ 760. issn: 17260604. doi: 10.1016/j.cirp.2016.05.004.

[30] K M Au and K M Yu. "A scaffolding architecture for conformal cooling design in rapid plastic injection moulding". In: The International Journal of Advanced Manufacturing Technology 34.56 (Sept. 2007), pp. 496-515. doi: 10.1007/s00170-006-0628-x.

[31] M A García, C García-Pando, and C Marto. "Conformal cooling in moulds with special geometry”. In: Innovative Developments in Virtual and Physical Prototyping - Proceedings of the 5th International Conference on Advanced Research and Rapid Prototyping. CRC Press, Sept. 2012, pp. 409-412. isbn: 9780415684187. doi: 10.1201/b11341-66.

[32] Yu Wang, Kai Min Yu, and Charlie C L Wang. "Spiral and conformal cooling in plastic injection molding”. In: CAD Computer Aided Design 63 (June 2015), pp. 1-11. issn: 00104485. doi: 10.1016/j.cad.2014.11.012.

[33] Antonio Armillotta, Raffaello Baraggi, and Simone Fasoli. "SLM tooling for die casting with conformal cooling channels". In: The International Journal of Advanced Manufacturing Technology 71.1-4 (Mar. 2014), pp. 573-583. doi: 10.1007/s00170-013-5523-7.

[34] Ferry P.W. Melchels et al. "Additive manufacturing of tissues and organs”. In: Progress in Polymer Science 37.8 (Aug. 2012), pp. 1079-1104. issn: 00796700. doi: 10.1016/j.progpolymsci. 2011.11.007. 
[35] S Biscaia et al. "Biofabrication of three-dimensional scaffolds of polycaprolactone with eggshell powder for bone regeneration". In: High Value Manufacturing: Advanced Research in Virtual and Rapid Prototyping. CRC Press, Sept. 2013, pp. 171-176. doi: 10.1201/b15961-32.

[36] Jayanthi Parthasarathy, Binil Starly, and Shivakumar Raman. "A design for the additive manufacture of functionally graded porous structures with tailored mechanical properties for biomedical applications". In: Journal of Manufacturing Processes 13.2 (Aug. 2011), pp. 160-170. issn: 15266125. doi: 10.1016/j.jmapro.2011.01.004.

[37] Xiaojian Wang et al. "Topological design and additive manufacturing of porous metals for bone scaffolds and orthopaedic implants: A review”. In: Biomaterials 83 (Mar. 2016), pp. 127-141. issn: 18785905. doi: 10.1016/j.biomaterials.2016.01.012.

[38] Tailored Fits AG. Tailored Fits AG. url: https://www.tailored-fits.com/en/.

[39] 3DPrint.com. Lowe's Offers New 3D Customization Options With Bespok3Designs Platform | 3DPrint.com | The Voice of 3D Printing / Additive Manufacturing. url: https://3dprint. com/147798/lowes-bespoke3designs/.

[40] Mohammad Vaezi et al. "Multiple material additive manufacturing - Part 1: A review". In: Virtual and Physical Prototyping. Vol. 8. 1. Taylor and Francis Group, Mar. 2013, pp. 19-50. doi: 10.1080/17452759.2013.778175.

[41] Autodesk. Within - Heat Exchange. url: http://withinlab.com/case-studies/index11. php (visited on 10/29/2017).

[42] Andrzej Grzesiak, Ralf Becker, and Alexander Verl. "The Bionic Handling Assistant: a success story of additive manufacturing". In: Assembly Automation 31.4 (2011), pp. 329-333. issn: 01445154. doi: 10.1108/01445151111172907.

[43] Digital Trends. Daimler starts 3D printing metal replacement parts for older Mercedes-Benz trucks. url: https://www.digitaltrends.com/cars/daimler-3d-printed-truckparts/.

[44] Daimler. Spare parts for trucks and buses made in a 3D printer | Daimler Sustainability Production. url: https://www.daimler.com/sustainability/production/3d-print.html.

[45] Sheng Yang and Yaoyao Fiona Zhao. Additive manufacturing-enabled design theory and methodology: a critical review. 2015. doi: 10.1007/s00170-015-6994-5.

[46] Joseph M Flynn et al. "Hybrid additive and subtractive machine tools - Research and industrial developments". In: International Journal of Machine Tools and Manufacture 101 (2015), pp. 79 101. doi: 10.1016/j.ijmachtools.2015.11.007.

[47] Rhys Jones et al. "RepRap - the replicating rapid prototyper". In: Robotica 29.01 (Jan. 2011), pp. 177-191. issn: 0263-5747. doi: 10.1017/S026357471000069X.

[48] Wessel W. Wits, J. Roberto Reyes García, and Juan M.Jauregui Becker. "How Additive Manufacturing Enables more Sustainable End-user Maintenance, Repair and Overhaul (MRO) Strategies”. In: Procedia CIRP. Vol. 40. 2016, pp. 694-699. doi: 10.1016/j.procir.2016.01. 156.

[49] Scientific United Nations Framework Convention on Climate Change United Nations Educationa and Cultural Organization. Action for Climate Empowerment. Guideline for accelerating solutions through education, training and public awareness. United Nations, 2016, pp. 1- 8. isbn: 9781100546476. url: http : / / unesdoc . unesco . org / images / 0024 / 002464 / 246435e.pdf. 
[50] Think3DPrint3D and Escher3D. Duet3D. url: https://www.duet3d.com/.

[51] Mr Beam. Mr Beam - Laser Cutting for Everybody. url: http://mr-beam.org/.

[52] DIYLILCNC. The DIYLILCNC Project. url: http://diylilenc.org/.

[53] Fabototum. FABtotum 3D Printing and CNC Company: Make Anything You Want. url: https: //www.fabtotum.com/.

[54] Circular Economy Portugal. Circular Economy Portugal - Repair Café. url: https://www. circulareconomy.pt/repaircafe. 